\title{
Solar Cell Made Using Graphene
}

\author{
T. Naveen Kumar
}

\begin{abstract}
The principal objective of this paper is to demonstrate the capability of graphene in making energy efficient solar panels inexpensively. Comparing to silicon solar cells, graphene solar cells are more efficient and provides double the power output in less time. Checking out the difference for the selection of elements between silicon and graphene, graphene leads with respect to properties like electrical conductivity, thermal conductivity, material strength and flexibility. The main difference is its inexpensiveness. Where there is increase in population and insufficient power production in villages, small scale industries, graphene solar panels will be installed to gain additional power without any problem .Researchers and industrialists quote graphene as the nearby future for power supply.
\end{abstract}

Keywords: conductivity,electrical,flexibility,graphene, inexpensiveness, panels, strength,thermal, solar, silicon cell

\section{Introduction}

Graphene is a wonder material with many superlatives to its name. It is the thinnest material in the universe and the strongest ever measured. Its charge carriers exhibit giant intrinsic mobility, have the smallest effective mass (it is zero) and can travel micrometer-long distances without scattering at room temperature. Graphene can sustain current densities 6 orders higher than copper, shows record thermal conductivity and stiffness, is impermeable to gases and reconciles such conflicting qualities as brittleness and ductility. Electron transport in graphene is described by a Dirac-like equation, which allows the investigation of relativistic quantum phenomena in a bench-top experiment.

What are other surprises that graphene keeps in store for us? This review analyses recent trends in graphene research and applications, and attempts to identify future directions in which the field is likely to develop. Regular graphene is a famously two-dimensional form of carbon just a molecule or so thick. To do so, they combined lithium oxide with carbon monoxide in a chemical reaction that forms lithium carbonate (Li2CO3) and the graphene. The Li2CO3 helps shape the graphene sheets and isolates them from each other, preventing the formation of garden-variety graphite. Furthermore, the Li2CO3 particles can be easily removed from 3D honeycomb-structured graphene by an acid.

The researchers determined that the graphene had excellent conductivity and high catalytic activity, raising the possibility that it could be used for energy storage and conversion. So they replaced the platinum counter electrode in a dye-sensitized solar cell with one made of the honeycomb graphene. Then they put the solar cell in the sunshine and measured its output. The cell with the graphene counter electrode converted $7.8 \%$ of the sun's energy into electricity, nearly as much as the conventional solar cell using costly platinum (8\%). The researchers note that the process of synthesizing the 3-D honeycomb graphene is relatively cheap and easy - there are no significant barriers to its wider adoption. As they put it: "Making it into a counter electrode posed no special challenges."

\section{The Occurrence Of Graphene}

Graphene is a rapidly rising star on the horizon of materials science and condensed matter physics. This strictly two- dimensional material exhibits exceptionally high crystal and electronic quality and, despite its short history, has already revealed a cornucopia of new physics and potential applications, which are briefly discussed here. Whereas one can be certain of the realness of applications only when commercial products appear, graphene no longer requires any further proof of its importance in terms of fundamental physics. Owing to its unusual electronic spectrum, graphene has led to the emergence of a new paradigm of "relativistic" condensed matter physics, where quantum relativistic phenomena, some of which are unobservable in high energy physics, can now be mimicked and tested in table-top experiments. More generally, graphene represents a conceptually new class of materials that are only one atom thick and, on this basis, offers new inroads into low-dimensional physics that has never ceased to surprise and continues to provide a fertile ground for applications. 


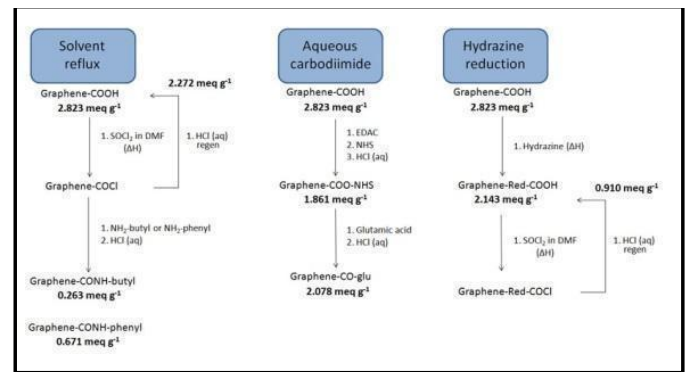

Fig 1

\section{The Structure Of Graphene}

Graphene is the name given to a flat monolayer of carbon atoms tightly packed into a two-dimensional (2D) honeycomb lattice, and is a basic building block for graphitic materials of all other dimensionalities. It can be wrapped up into 0D fullerenes, rolled into 1D nanotubes or stacked into 3D graphite. Theoretically, graphene (or "2D graphite") has been studied for sixty years1-3 and widely used for describing properties of various carbon-based materials. Forty years later, it was realized that graphene also provides an excellent condensedmatter analogue of (2+1)-dimensional quantum electrodynamics4-6, which propelled graphene into a thriving theoretical toy model. On the other hand, although known as integral part of 3D materials, graphene was presumed not to exist in the free state, being described as an "academic" material5 and believed to be unstable with respect to the formation of curved structures such as soot, fullerenes and nanotubes. All of a sudden, the vintage model turned into reality, when free-standing graphene was unexpectedly found three years ago7,8 and, especially, when the follow-up experiments9,10 confirmed that its charge carriers were indeed massless Dirac fermions. So, the graphene "gold rush" has begun.

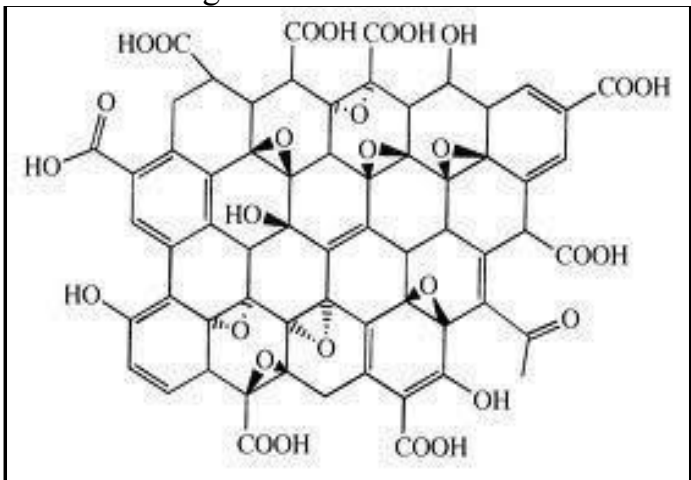

Fig 2.1

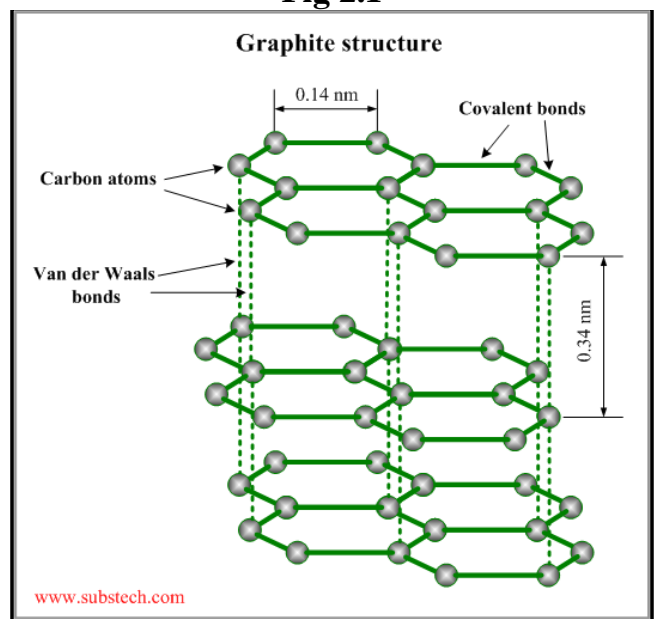

Fig 2.2

\section{Brief History Of Graphene}

Before reviewing the earlier work on graphene, it is useful to define what 2D crystals are. Obviously, a single atomic plane is a 2D crystal, whereas 100 layers should be considered as a thin film of a 3D material. But how many layers are needed to make a 3D structure? For the case of graphene, the situation has recently become reasonably clear. It was shown that the electronic structure rapidly evolves with the number of layers, 
approaching the 3D limit of graphite already at 10 layers20. Moreover, only graphene and, to a good approximation, its bilayer have simple electronic spectra: they are both zero-gap semiconductors (can also be referred to as zero-overlap semimetals) with one type of electrons and one type of holes. For 3 and more layers, the spectra become increasingly complicated: Several charge carriers appear7,21, and the conduction and valence bands start notably overlapping7,20. This allows one to distinguish between single-, double- and few- (3 to $<10$ ) layer graphene as three different types of 2D crystals ("graphenes"). Thicker structures should be considered, to all intents and purposes, as thin films of graphite. From the experimental point of view, such a definition is also sensible. The screening length in graphite is only $\approx 5 \AA$ (that is, less than 2 layers in thickness) 21 and, hence, one must differentiate between the surface and the bulk even for films as thin as 5 layers.21,22 Earlier attempts to isolate graphene concentrated on chemical exfoliation. To this end, bulk graphite was first intercalated (to stage I) 23 so that graphene planes became separated by layers of intervening atoms or molecules. This usually resulted in new 3D materials23. However, in certain cases, large molecules could be inserted between atomic planes, providing greater separation such that the resulting compounds could be considered as isolated graphene layers embedded in a 3D matrix. Furthermore, one can often get rid of intercalating molecules in a chemical reaction to obtain a sludge consisting of restacked and scrolled graphene sheets24-26. Because of its uncontrollable character, graphitic sludge has so far attracted only limited interest. There have also been a small number of attempts to grow graphene. The same approach as generally used for growth of carbon nanotubes so far allowed graphite films only thicker than $\approx 100$ layers 27 . On the other hand, single- and few-layer graphene have been grown epitaxially by chemical vapour deposition of hydrocarbons on metal substrates28,29 and by thermal decomposition of SiC30-34. Such films were studied by surface science techniques, and their quality and continuity remained unknown. Only lately, few-layer graphene obtained on $\mathrm{SiC}$ was characterized with respect to its electronic properties, revealing high-mobility charge carriers 32,33 . Epitaxial growth of graphene offers probably the only viable route towards electronic applications and, with so much at stake, a rapid progress in this direction is expected. The approach that seems promising but has not been attempted yet is the use of the previously demonstrated epitaxy on catalytic surfaces 28,29 (such as Ni or Pt) followed by the deposition of an insulating support on top of graphene and chemical removal of the primary metallic substrate.

\section{Conductivity Without Charge Carriers}

Another important observation is that graphene's zero-field conductivity $\sigma$ does not disappear in the limit of vanishing $\mathrm{n}$ but instead exhibits values close to the conductivity quantum $\mathrm{e} 2 / \mathrm{h}$ per carrier . It shows the lowest conductivity $\sigma$ min measured near the neutrality point for nearly 50 single-layer devices. For all other known materials, such a low conductivity unavoidably leads to a metal-insulator transition at low $\mathrm{T}$ but no sign of the transition has been observed in graphene down to liquid-helium T. Moreover, although it is the persistence of the metallic state with $\sigma$ of the order of $\mathrm{e} 2 / \mathrm{h}$ that is most exceptional and counterintuitive, a relatively small spread of the observed conductivity values (see Fig. 5) also allows one to speculate about the quantization of omin. We emphasize that it is the resistivity (conductivity) that is quantized in graphene, in contrast to the resistance (conductance) quantization known in many other transport phenomena. Minimum quantum conductivity has been predicted for Dirac fermions by a number of theories5,44,45,47,60-64. Some of them rely on a vanishing density of states at zero $\mathrm{E}$ for the linear 2D spectrum. However, comparison between the experimental behaviour of massless and massive Dirac fermions in graphene and its bilayer allows one to distinguish between chirality- and masslessness- related effects. To this end, bilayer graphene also exhibits a minimum conductivity of the order of e2/h per carrier type,55,65 which indicates that it is chirality, rather than the linear spectrum, that is more important. Most theories suggest $\sigma \mathrm{min}=4 \mathrm{e} 2 / \mathrm{h} \pi$, which is of about $\pi$ times smaller than the typical values observed experimentally. One can see in Fig. 5 that the experimental data do not approach this theoretical value and mostly cluster around $\sigma \mathrm{min}=4 \mathrm{e} 2 / \mathrm{h}$ (except for one low- $\mu$ sample that is rather unusual by also exhibiting 100\%-normal weak localization behaviour at high n; see below). This disagreement has become known as "the mystery of a missing pie", and it remains unclear whether it is due to theoretical approximations about electron scatteringin graphene or because the experiments probed only a limited range of possible sample parameters (e.g.,length-to-width ratios47). To this end, note that close to the neutrality point $(\mathrm{n} \leq 1011 \mathrm{~cm}-2)$ graphene islikely to conduct as a random network of electron and hole puddles (A.K.G. \& K.S.N. unpublished). Such microscopic inhomogeneity is probably inherent to graphene (because of graphene sheet's warping/rippling)18,66 but so far has not been taken into account by theory. Furthermore, macroscopic inhomogeneity (on the scale larger than the mean free path 1) also plays an important role in measurements of $\sigma \mathrm{min}$. The latter inhomogeneity can explain a high- $\sigma$ tail in the data scatter in Fig. 5 by the fact that $\sigma$ reached its lowest values at slightly different $\mathrm{Vg}$ in different parts of a sample, which yields effectively higher values of experimentally measured $\sigma \mathrm{min}$. 


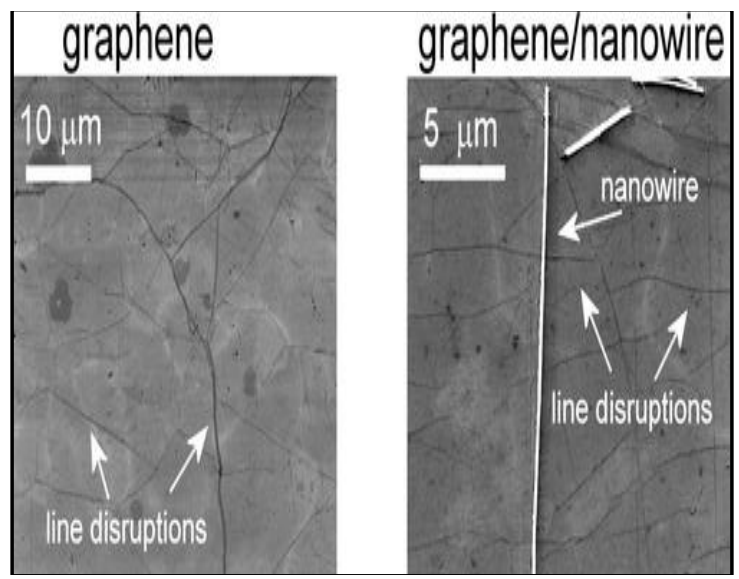

Fig 3: Polycrystalline graphine

Polycrystalline graphene grown by chemical vapor deposition(CVD) on metals and transferred onto arbitrary substrates has line defects and disruptions such as wrinkles,ripples and folding that adversely affect graphene transport properties through the scaterring of the charge carriers.it is found that graphene assembled with metal nanowires(Nws) dramatically reduces the resistance of graphene films. Graphene/NWs films with a sheet resistance comparableyo that of the intrinsic resistance of graphene have been obtained ad tested as a transparent electrode replacing indium tin oxide films in ElectroChromic(EC) devices. The successful integration of such graphene?NW films into EC devices demonstrates their potential for a wide range of opto electric device applications.

\section{Graphene Dreams}

Despite the reigning optimism about graphene-based electronics, "graphenium" microprocessors are unlikely toappear for the next 20 years. In the meantime, one can certainly hope for many other graphene-based applications to come of age. In this respect, clear parallels with nanotubes allow a highly educated guess of whatto expect soon.

The most immediate application for graphene is probably its use in composite materials. Indeed, it has been demonstrated that a graphene powder of uncoagulated micron-size crystallites can be produced in a way scaleable to mass production 17. This allows conductive plastics at less than 1 volume percept filling 17, which in combination with low production costs makes graphene-based composite materials attractive for a variety of uses. However, it seems doubtful that such composites can match the mechanical strength of their nanotube counterparts because of much stronger entanglement in the latter case.

Another enticing possibility is the use of graphene powder in electric batteries that are already one of the main markets for graphite. An ultimately large surface-to-volume ratio and high conductivity provided by Graphene powder can lead to improvements in batteries' efficiency, taking over from carbon nanofibres used in modern batteries. Carbon nanotubes have also been considered for this application but graphene powder has an important advantage of being cheap to produce17.

One of the most promising applications for nanotubes is field emitters and, although there have been no reports yet about such use of graphene, thin graphite flakes were used in plasma displays (commercial prototypes) long before graphene was isolated, and many patents were filed on this subject. It is likely that graphene powder can offer even more superior emitting properties. Carbon nanotubes were reported to be an excellent material for solid-state gas sensors but graphene offers clear advantages in this particular direction 41. Spin-valve and superconducting field-effect transistors are also obvious research targets, and recent reports describing a hysteretic magnetoresistance90 and substantial bipolar supercurrents91 prove graphene's major potential for these applications. An extremely weak spin-orbit coupling and the absence of hyperfine interaction in 12C-graphene make it an excellent if not ideal material for making spin qubits. This guarantees graphenebased quantum computation to become an active research area. Finally, we cannot omit mentioning hydrogen storage, which has been an active but controversial subject for nanotubes. It has already been suggested that graphene is capable of absorbing an ultimately large amount of hydrogen92, and experimental efforts in this direction are duly expected. 


\section{After The Gold Rush}

It has been just over 2 years since graphene was first reported and, despite remarkably rapid progress, only the very tip of the iceberg has been uncovered so far. Because of the short time scale, most experimental groups working now on graphene have not published even a single paper on the subject, which has been a truly frustrating experience for theorists. This is to say that, at this time, no review can possibly be complete. Nevertheless, the research directions explained or pencilled here should persuade even die-hard sceptics that graphene is not a fleeting fashion but is here to stay, bringing up both more exciting physics and, perhaps, even wide-ranging applications.

\section{Some properties of graphene}

\subsection{Density of graphene}

The unit hexagonal cell of graphene contains two carbon atoms and has an area of $0.052 \mathrm{~nm} 2$. We can thus calculate its density as being $0.77 \mathrm{mg} / \mathrm{m} 2$. A hypothetical hammock measuring $1 \mathrm{~m} 2$ made from graphene would thus weigh $0.77 \mathrm{mg}$.

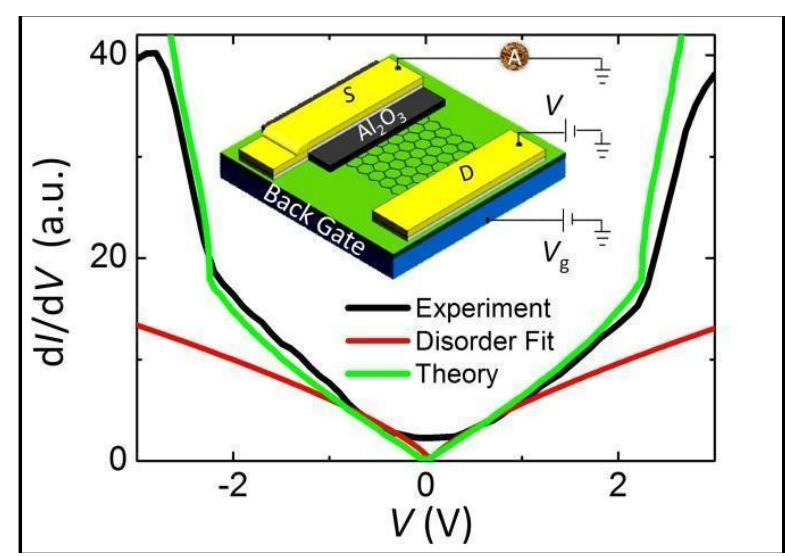

Fig 4.1: Density of states of reduced graphene oxides

\subsection{Optical transparency of graphene}

Graphene is almost transparent, it absorbs only $2.3 \%$ of the light intensity, independent of the wavelength in the optical domain. This number is given by $\pi \alpha$, where $\alpha$ is the fine structure constant. Thus suspended graphene does not have any color.

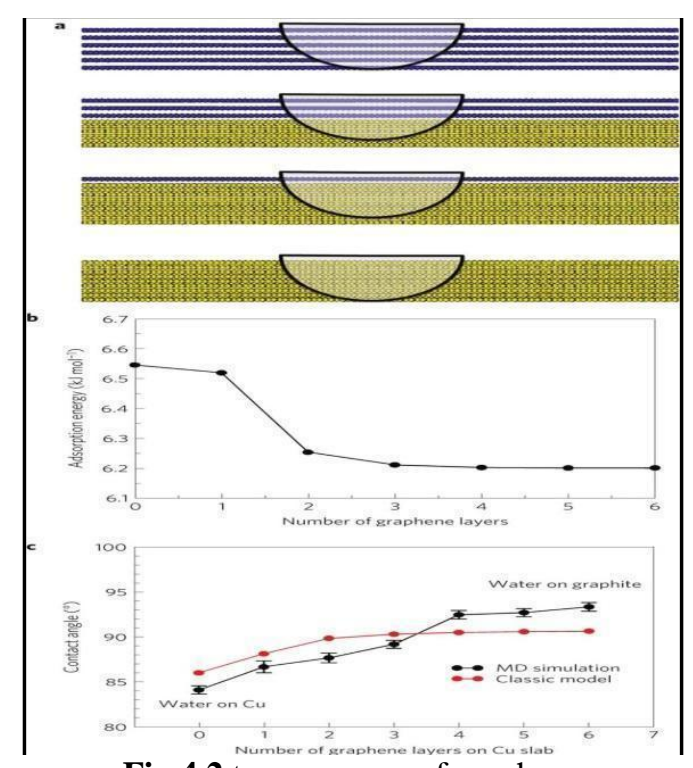

Fig 4.2 transparency of graphene

\subsection{Strength of graphene}

Graphene has a breaking strength of $42 \mathrm{~N} / \mathrm{m}$. Steel has a breaking strength in the range of $250-1200$ $\mathrm{MPa}=0.25-1.2 \times 109 \mathrm{~N} / \mathrm{m} 2$. For a hypothetical steel film of the same thickness as graphene (which can be taken to be $3.35 \AA=3.35 \times 10-10 \mathrm{~m}$, i.e. the layer thickness in graphite), this would give a $2 \mathrm{D}$ breaking strength of 0.084 $0.40 \mathrm{~N} / \mathrm{m}$. Thus graphene is more than 100 times stronger than the strongest steel. 
In our $1 \mathrm{~m} 2$ hammock tied between two trees you could place a weight of approximately $4 \mathrm{~kg}$ before it would break. It should thus be possible to make an almost invisible hammock out of graphene that could hold a cat without breaking. The hammock would weigh less than one $\mathrm{mg}$, corresponding to the weight of one of the cat's whiskers.

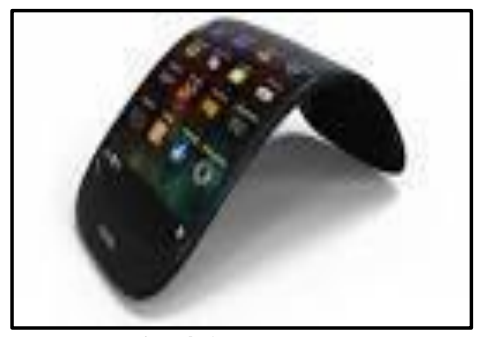

Fig 4.3 flexibility

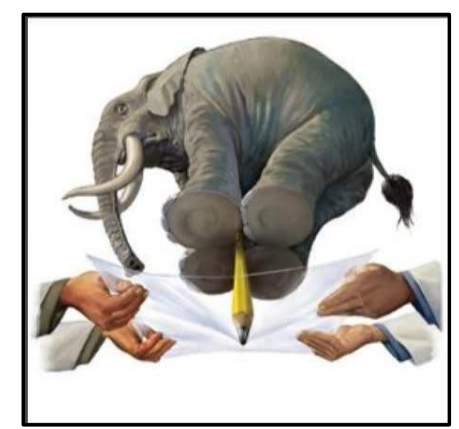

Fig 4.4 Material strength

\subsection{Electrical conductivity of graphene}

The sheet conductivity of a 2D material is given by . The mobility is theoretically limited to $\mu=200,000$ $\mathrm{cm} 2 \mathrm{~V}-1 \mathrm{~s}-1$ by acoustic phonons at a carrier density of $\mathrm{n}=1012 \mathrm{~cm}-2$. The $2 \mathrm{D}$ sheet resistivity, also called the resistance

per

Square, is then $31 \Omega$. Our fictional hammock measuring $1 \mathrm{~m} 2$ would thus have a resistance of $31 \Omega . \sigma=\mathrm{en} \mu$

Using the layer thickness we get a bulk conductivity of $0.96 \times 106 \Omega-1 \mathrm{~cm}-1$ for graphene. This is somewhat higher than the conductivity of copper which is $0.60 \times 106 \Omega-1 \mathrm{~cm}-1$.

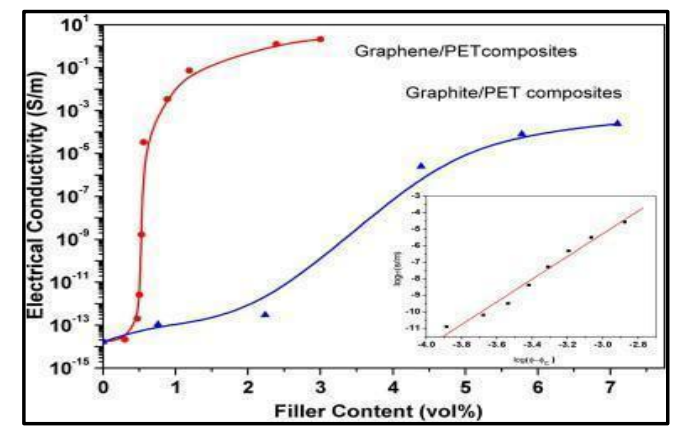

Fig 4.5

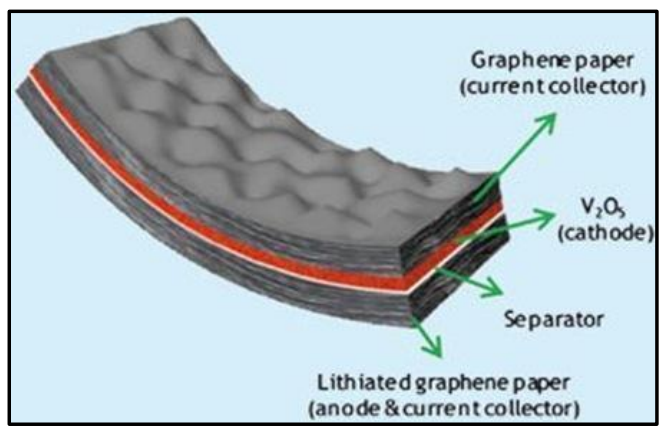

Fig 4.6 


\subsection{Thermal conductivity}

The thermal conductivity of graphene is dominated by phonons and has been measured to be approximately $5000 \mathrm{Wm}-1 \mathrm{~K}-1$. Copper at room temperature has a thermal conductivity of $401 \mathrm{Wm}-1 \mathrm{~K}-1$. Thus graphene conducts heat 10 times better than copper.

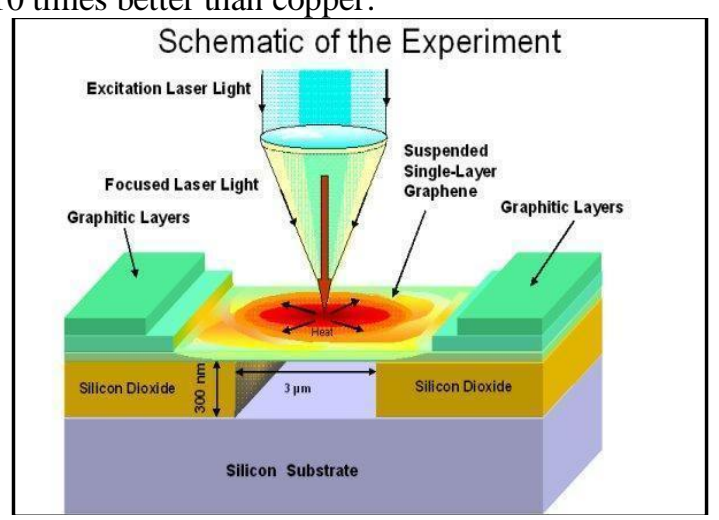

Fig 4.7

\section{Chemistry Matters}

Graphene is an ultimate incarnation of the surface: It has two faces with no bulk left in between. While this surface's physics is currently at the center of attention, its chemistry remains largely unexplored. What we have so far learned about graphene chemistry is that, similar to the surface of graphite, graphene can adsorb and desorb various atoms and molecules (for example, $\mathrm{NO} 2, \mathrm{NH} 3, \mathrm{~K}$ and $\mathrm{OH}$ ). Weakly attached adsorbates often act as donors or acceptors and lead to changes mostly in the carrier concentration so that graphene remains highly conductive (29). Other adsorbates like $\mathrm{H}+$ or $\mathrm{OH}-$ give rise to localized ("mid-gap") states close to NP, which results in poorly conductive derivatives such as graphene oxide (6) and "single-sided graphane" (30). Despite the new names, these are not new chemical compounds but the same graphene randomly decorated with adsorbates. Thermal annealing or chemical treatment allows the reduction of graphene to its original state with relatively few defects left behind (30). This reversible dressing up and down is possible due to the robust atomic scaffold that remains intact during chemical reactions. Within this surface science perspective, graphene chemistry looks similar to that of graphite, and the latter can be used for guidance. There are principal differences too. First, chemically-induced changes in graphene's properties are much more pronounced because of the absence of an obscuring contribution from the bulk (29). Second, unlike graphite's surface, graphene is not flat, but typically exhibits nm-scale corrugations (8). The associated strain and curvature can markedly influence local reactivity. Third, reagents can attach to both graphene faces, and this alters the energetics allowing chemical bonds that would be unstable if only one surface were exposed (31). An alternative to the surface chemistry perspective is to consider graphene as a giant flat molecule (as first suggested by Linus Pauling). As any other molecule, graphene can partake in chemical reactions. The important difference between the two viewpoints is that in the latter case adsorbates are implicitly assumed to attach to the carbon scaffold in a stoichiometric manner, that is, periodically rather than randomly. This should result in new 2D crystals with distinct electronic structures and different electrical, optical, chemical, etc. properties. The first known example is graphane, a 2D hydrocarbon with one hydrogen atom attached to every site of the honeycomb lattice $(30,31)$. Many other graphene-based crystals should be possible because adsorbates are likely to self-organize into periodic structures, similar to the case of graphite well known for its surface superstructures. Instead of doping with atomic hydrogen as in graphane, $\mathrm{F}-, \mathrm{OH}-$ and many functional groups look viable candidates in the search for novel graphene based 2D crystals. Graphene chemistry is likely to play an increasingly important role in future developments. For example, stoichiometric derivatives offer a way to control the electronic structure, which is of interest for many applications including electronics. Chemical changes can probably be induced even locally. Imagine then an all-graphene circuitry with interconnects made from pristine graphene whereas other areas are modified to become semiconducting and allow transistors. Disordered graphene-based derivatives should not be overlooked either. They can probably be referred to as functionalized graphene so that it is suitable for specific applications. "Graphene paper" is a spectacular example of how important such functionalization could be (Fig. 3). If it is made starting with a suspension of nonfunctionalized flakes (5), the resulting material is porous and extremely fragile and can float in air like a feather. However, the same paper made of graphene oxide is dense, stiff and strong $(6,32)$. In the latter case, the functional groups bind individual sheets together, which results in a microscopic structure not dissimilar to that of nacre known for its strength. Instead of aragonite bound in nacre by biopolymer glue, graphene oxide laminate and, especially, its reduced version (32) make use of atomic-scale stitching of the strongest known nanomaterial. 


\section{Sitting on a Graphene Mine}

There has been an explosion of ideas that suggest graphene for virtually every feasible use. This is often led by analogies with carbon nanotubes that continue to serve as a guide in searching for new applications. For example, graphene powder is considered to be excellent filler for composite materials (6). Reports have also been made on graphene-based supercapacitors, batteries and field emitters, but it is too early to say whether graphene is able to compete with the other materials, including nanotubes. Less expectedly, graphene has emerged as a viable candidate for the use in optoelectronics $(10,39)$. Suspensions offer an inexpensive way for making graphene-based coatings by spinning or spraying (Fig. 1B). An alternative is the transfer of films grown on $\mathrm{Ni}(9,10)$. These coatings are often suggested as a competitor for indium tin oxide (ITO) that is the industry standard in such products as solar cells, LCD displays, etc. However, graphene films exhibit resistivity of several hundred Ohms for the standard transparency of $\sim 80 \%(9,10,39)$. This is 2 orders higher than for ITO and unacceptable in many applications (for example, solar cells).

It remains to be seen whether the conductivity can be improved to the required extent. Having said that, grapheme coatings also offer certain advantages over ITO. They are chemically stable, robust and flexible and can even be folded, which gives them a good chance of beating the competition in touch screens and bendable applications.

There is also fast growing interest in graphene as a base material for nanoelectromechanical systems (NEMS) $(32,40)$.

Graphene is ultimately light and stiff, which are the essential characteristics sought in NEMS for sensing applications.

Graphene-based resonators offer low inertial masses, ultra-high frequencies and, in comparison with nanotubes, lowresistance contacts that are essential for matching the impedance of external circuits. Graphene membranes have so far shown quality factors of $\sim 100$ at $100 \mathrm{MHz}$ frequencies (40). Even more encouraging are data for drum resonators made from reduced graphene oxide films (32). These nm-thick polycrystalline NEMS (Fig. 4C) exhibit high Young's moduli (comparable to those of graphene) and quality factors of $\sim 4,000$ at room T. Importantly, the films can be produced as wafers and then processed by standard microfabrication techniques. Further developments (increasing the frequency and improving quality factors) should allow graphene NEMS to assail such tantalizing challenges as inertial sensing of individual atoms and the detection of zero-point oscillations. Among other applications that require mentioning are labs-on-chips (electronic noses) and various resistive memories. The high sensitivity of graphene to its chemical environment is well acknowledged, after sensors capable of detecting individual gas molecules were demonstrated (29). The prospect of graphene wafers has put this curiosity-driven record into another perspective. Imagine an array of graphene devices each functionalized differently to be able to react to different chemicals or bio-molecules. Such functionalization has been intensively researched for the case of carbon nanotubes, and graphene adds the possibility of massproduced arrays of identical devices. Furthermore, there are several enticing reports on non-volatile memories in which graphene-based wires undergo reversible resistance switching by, for example, applying a sequence of current pulses $(41,42)$. The underlying mechanism remains largely unknown but such $\mathrm{nm}$-scale switches present an attractive alternative to phase-change memories and deserve further attention. Reports on grapheneferroelectric memories (43) are also encouraging, especially due to the basic simplicity of their operation.

\section{CVD Graphene: electronic properties and applications}

$\begin{array}{ccccc}\text { Purdue } & \text { University, } & \text { West } & \text { Lafayette, } & \text { Indiana, } \\ \text { Chemical vapor deposition } & \text { (CVD) has emerged as one of the most }\end{array}$ promising methods for scalable production of graphene suitable for a wide range of experimental studies and practical applications. We have studied electronic properties of large scale polycrystalline graphene as well as graphene single crystals synthesized by ambient-pressure CVD on Cu.The graphene can be transferred to appropriate substrates for transport measurements and device applications. We show that such CVDsynthetic graphene is intrinsic graphene by observing the characteristic halfinteger quantum Hall effect (QHE), even in graphene films as large as center-meters in size, demonstrating their excellent quality and electronic homogeneity. Wehave also characterized electronic scattering at individual grain boundaries and graphene edges by transport measurements, STM and Raman mapping. Examples of potential applications of CVD Graphene in quantum metrology, molecular electronics and radiation detection will be discussed 


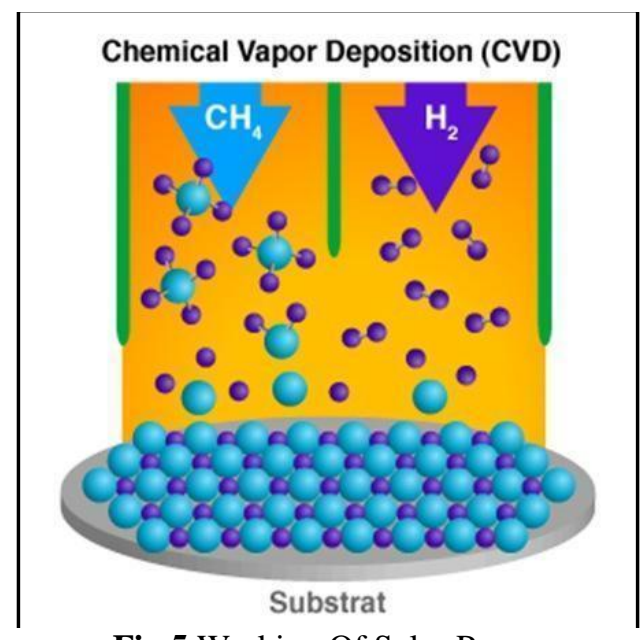

Fig 5 Working Of Solar Power

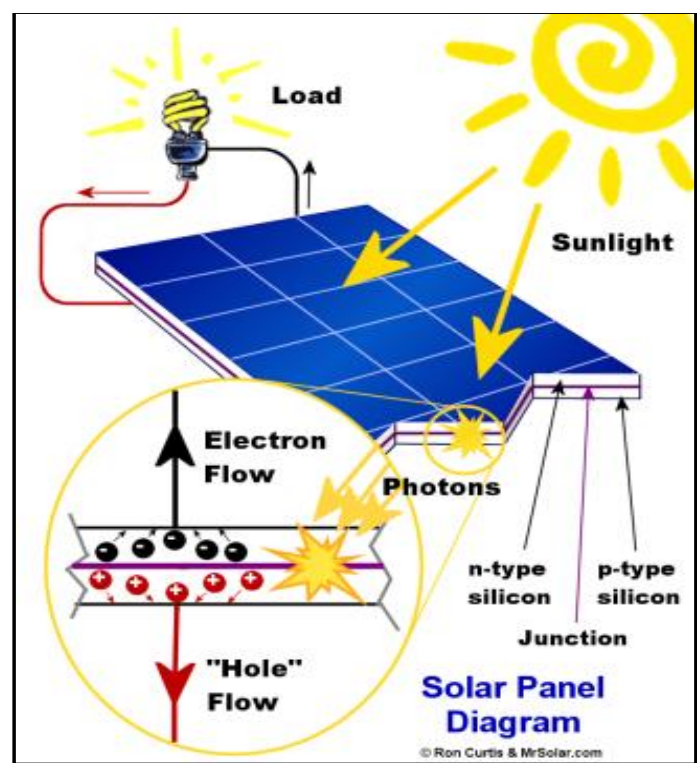

Fig 6 Working Of Graphene Solar Cells

Dye-sensitized solar cells are thin, flexible, easy to make and very good at turning sunshine into electricity. However, a key ingredient is one of the most expensive metals on the planet: platinum. While only small amounts are needed, at $\$ 1,500$ an ounce, the cost of the silvery metal is still significant.

Yun Hang $\mathrm{Hu}$, the Charles and Caroll McArthur Professor of Materials Science and Engineering, has developed a new, inexpensive material that could replace the platinum in solar cells without degrading their efficiency: 3D graphene.

Regular graphene is a famously two-dimensional form of carbon just a molecule or so thick. Hu and his team invented a novel approach to synthesize a unique 3D version with a honeycomb like structure. To do so, they combined lithium oxide with carbon monoxide in a chemical reaction that forms lithium carbonate $\left(\mathrm{Li}_{2} \mathrm{CO}_{3}\right)$ and the honeycomb graphene. The $\mathrm{Li}_{2} \mathrm{CO}_{3}$ helps shape the graphene sheets and isolates them from each other, preventing the formation of garden-variety graphite. Furthermore, the Li2CO3 particles can be easily removed from 3D honeycomb-structured graphene by an acid.

The researchers determined that the 3D honeycomb graphene had excellent conductivity and high catalytic activity raising the possibility that it could be used for energy storage and conversion. So they replaced the platinum counter electrode in a dye-sensitized solar cell with one made of the 3D honeycomb graphene. Then they put the solar cell in the sunshine and measured its output.

The cell with the 3D graphene counter electrode converted 7.8 percent of the sun's energy into electricity, nearly as much as the conventional solar cell using costly platinum (8 percent).

Synthesizing the 3D honeycomb graphene is neither expensive nor difficult, said $\mathrm{Hu}$, and making it into a counter electrode posed no special challenges. 
The article describing the work, "3D Honeycomb-Like Structured Graphene and Its High Efficiency as a Counter-Electrode Catalyst for Dye-Sensitized Solar Cells," coauthored by Hu, Michigan Tech graduate student Hui Wang, Franklin Tao of the University of Notre Dame, Dario J. Stacchiola of Brookhaven National Laboratory and Kai Sun of the University of Michigan, was published online July 29 in the journal Angewandte Chemie International Edition.

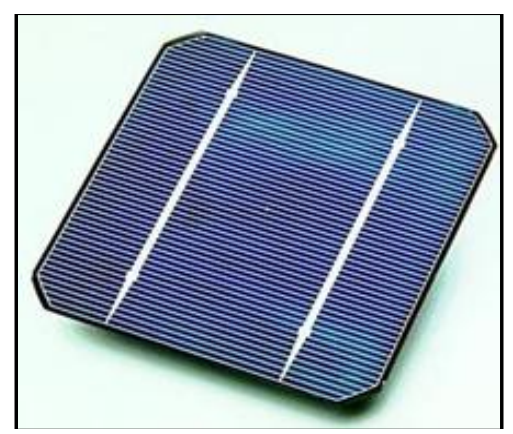

Fig 7 Graphene solar cell

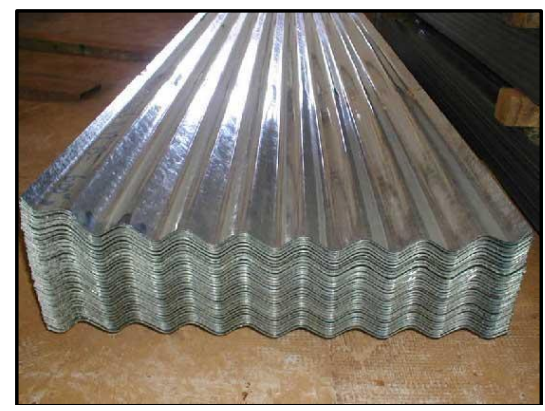

Graphene sheets

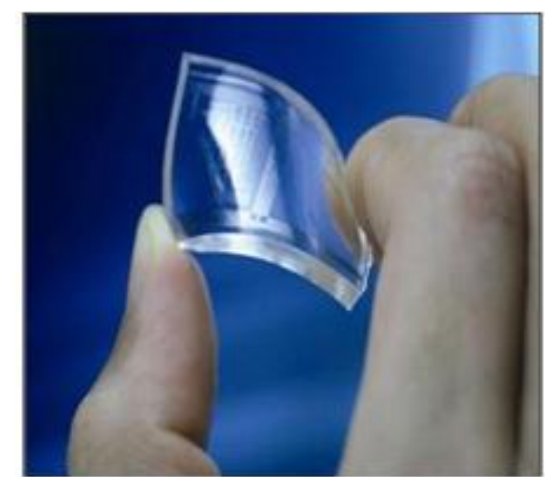

Fig 8 Graphene Film(unbreakable)

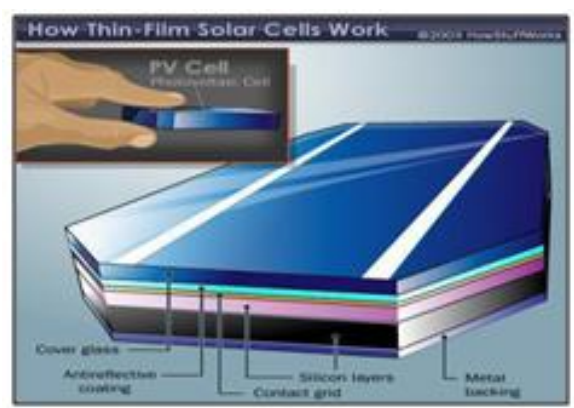

Fig 9 Graphene layers are introduced in place of silicon layers 


\section{Conclusion}

The paper explains and demonstrates the use of Graphene for making solar panels for deriving extreme power supply using the unlimited solar energy. Listing and comparing the properties of the silicon and graphene, graphene leads with respect to electrical conductivity, thermal conductivity, flexibility ,material strength. The main reason for selection of graphene is its inexpensiveness .Also there are a number of ways of preparation of graphene films, one of the main process is chemical vapor deposition(CVD). Graphene is said to replace electronics and electricity in nearby future.

\section{References}

[1]. Yong-Tae Kim, Department of Chemistry, BK-21 School of Chemical Materials Science SKKU Advanced Institute of Nanotechnology, Sungkyunkwon University, Swon, Korea

[2]. Byung Hee Hong, Department of Chemistry, Seoul National University, Seoul, Korea

[3]. Daeheon Choi, Department of Chemistry \& Institute for Advanced Research, Nagoya University, Nagoya 464 - 8620 Japan.

[4]. Yasuo Azuma,Materials and Structures Laboratory, Tokyo Institute of Technology, Yokohama 226-8503,Japan

[5]. Ting YU,Division of Physics and Applied, School of Physical and Mathematical Sciences, Nanyang Technological University, Singapore

[6]. Riichiro Saito,Department of Physics, Tohoku University, Sendai, Miyagi 9808578, Japan

[7]. Websites:www.google.com,www.wikepedia.com.

[8]. Book:Alternate energy:Assesment and Implementation,James J Winebrake

[9]. Book:Designing with Solar Power:building integrated Photovoltaics,Deo Prasad,Mark Snow 